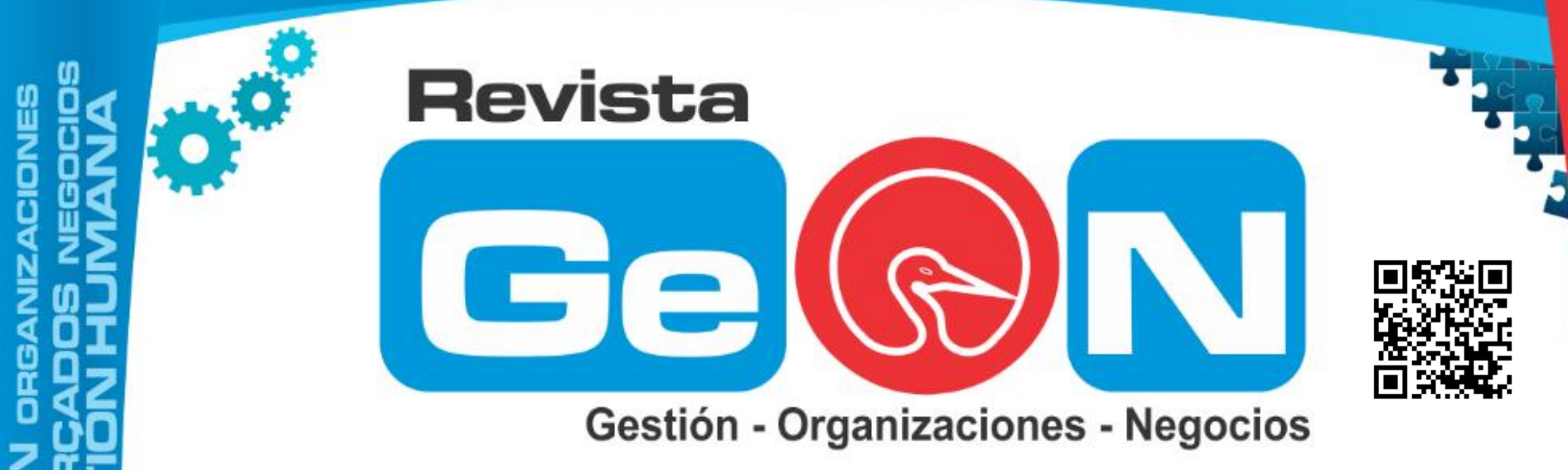

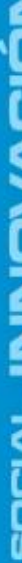

(1)

ISEN 204E - 3970

Volumen 11 Número 0 Diciembre Jullo 14

Revista Electrónica de la Facultad de Ciencias Económicas de la Universidad de los Llanos

$4 \leq 1$

$3<$

$2 \frac{1}{2} \frac{1}{4}$
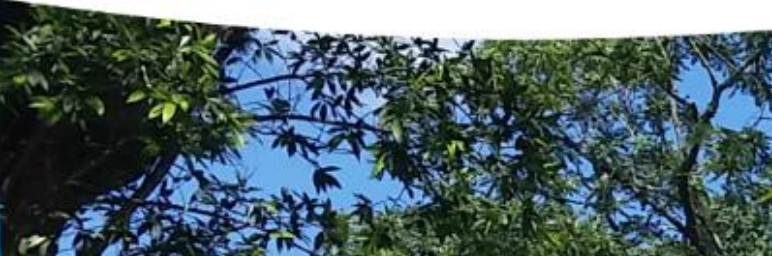

(1)

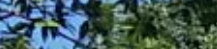

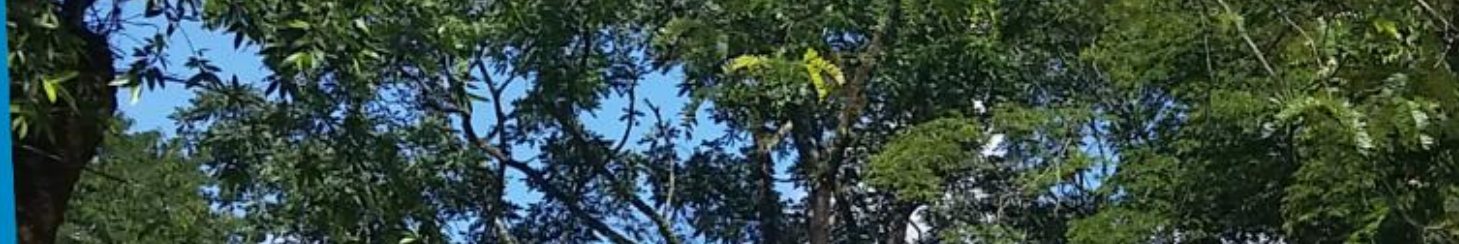

다

(11) को

$2 \frac{1}{0}$

i c

iii $\frac{2}{11}$ if

in

402

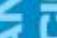

40

20 III

iㅣㄴ

12

10

10

ii 1

U1

$2 \frac{1}{2}$

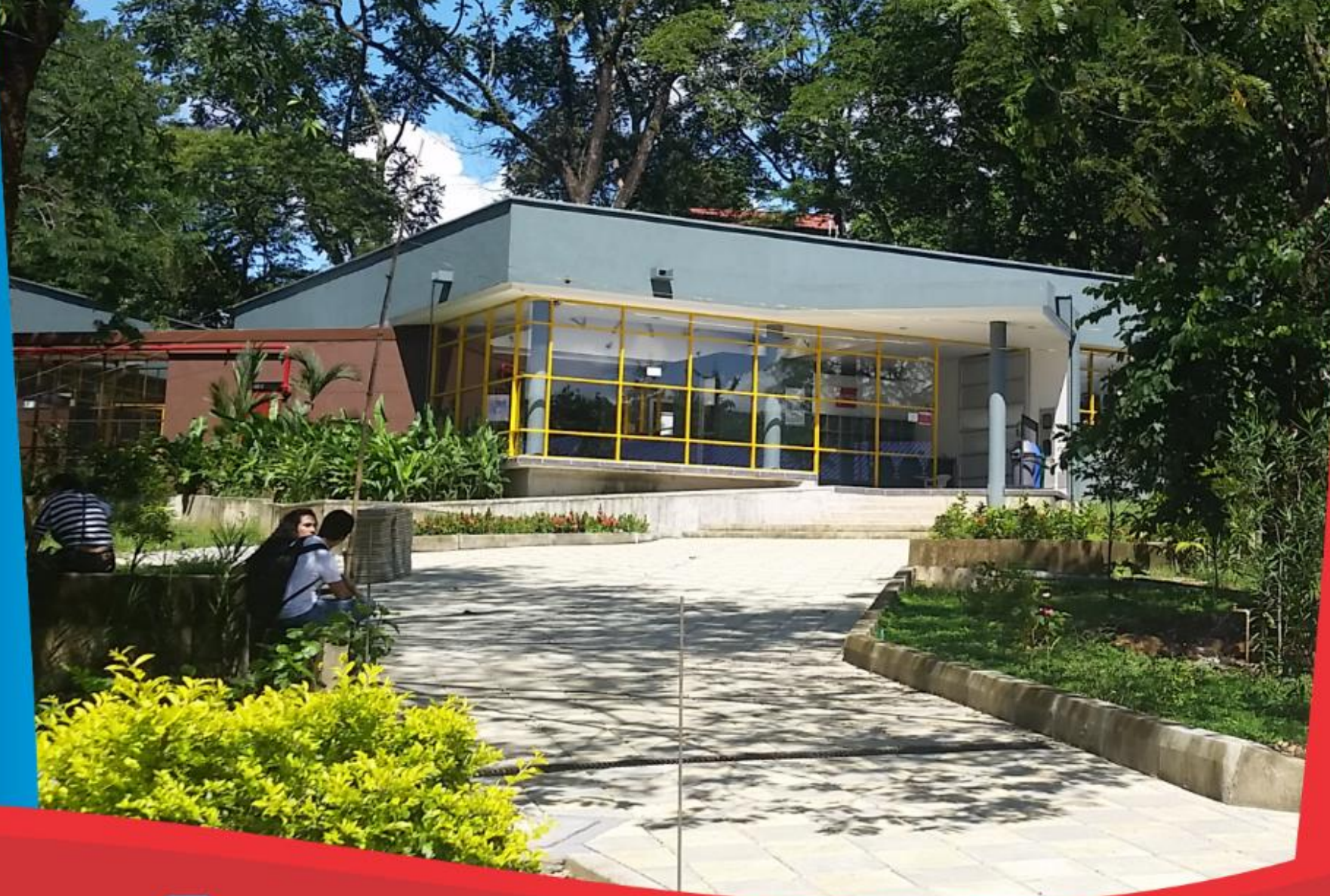

I/revistaGEON @RevistaGeon

http://revistageon.unillanos.edu.co 


\section{El software libre como una herramienta para el emprendimiento de base tecnológica en el departamento del Meta - por Jorge García y María Paula Estupiñan}

Jorge García Álvarez

Profesional en Mercadeo y Publicidad

Especialista en Alta Gerencia

Candidato Magister en Administración de Empresas

Universidad Nacional de Colombia

@ jorgegarciamyp

María Paula Estupiñan Tiuso

Administradora de Empresas

Especialista en Administración y Negocios

Candidato Magister en Administración de Empresas

Universidad Nacional de Colombia

@ paulaestupinan

\section{RESUMEN}

El uso de software libre en diferentes sectores productivos, ha generado una gran oportunidad para las empresas para adaptar los modelos y políticas de negocio en los entornos de desarrollo tecnológico y competitividad empresarial, con el fin de mejorar su productividad y lograr ventajas competitivas que le permitan interactuar efectivamente en el mercado nacional e internacional.

Sin embargo, el Estado atendiendo a las recomendaciones dispuestas por la UNESCO, en donde se promueve al software libre por impulsar a la denominada revolución de conocimiento abierto, ha cambiado los paradigmas de crecimiento de la mayoría de sectores empresariales. (Escalante, 2009)

Esta afirmación, es evidenciada por Rappa(2001) y Rayport(1999) al indicar que debido a la variedad de herramientas tecnológicas para el desarrollo de software y al no existir esquemas de producción orientados al software libre, no se han definido las condiciones de los modelos de negocio existentes, por lo cual, empresas dedicadas a esta actividad desconocen oportunidades en la industria nacional del software libre (INSL).

Según Escalante \& García, los modelos de negocios derivados de la transformación en los entornos de desarrollo de aplicaciones, se considera uno de los temas de mayor importancia para el sector empresarial, debido a que los nuevos desarrollos bajo código abierto han transformado la forma tradicional de hacer negocios, incluso clasificándolo en un modelo no comercial aún en estudio; sin embargo, la verdadera preocupación del sector industrial del software es la poca evidencia del cómo se ha transformado y las acciones que deben tomarse al respecto.

PALABRAS CLAVES: Software libre, Competitividad, emprendimiento, Productividad.

\section{ABSTRACT}

The use of free software in different productive sectors, has generated a great opportunity for companies to adapt business models and policies in the environments of technological development and competitiveness, in order to improve productivity and achieve competitive advantages that allow them interact effectively in national and international market.

However, the State following the recommendations set forth by UNESCO, where it promotes free software to boost the so-called open knowledge revolution has changed the paradigms of growth in most business sectors. (Escalante, 2009)

This statement is evidenced by Rappa (2001) and Rayport (1999) to indicate that due to the variety of technological tools for software development and the absence of production schemes oriented free software, no defined conditions of existing business models, therefore, companies engaged in this activity unknown opportunities in the domestic industry of free software (INSL).

According Escalante \& García, business models from the processing in the application development environment, is considered one of the most important issues for the business sector, because new developments in open source have transformed the traditional way do business even classifying non commercial model still under study; however, the real concern of the software industry is the evidence of how little has changed and the actions to be taken in this regard.

KEYWORDS: Free Software, competitiveness, entrepreneurship, Productivity. 


\section{METODOLOGÍA.}

La presente investigación tiene como objetivo identificar el nivel de uso del software libre como una herramienta para el emprendimiento tecnológico en el Departamento del Meta, a partir de una metodología de investigación exploratoria y descriptiva, la cual se encuentra fundamentada en la estrategia de diseño de campo; La población objeto de estudio está compuesta por empresas dedicadas a la prestación de servicios tecnológicos y desarrollo de productos de base tecnológica agrupados en un clúster tecnológico importante de la región, donde se concentran 11 emprendimientos con el fin comercial mencionado. Mediante un focus group se contactaron 5 empresas que han desarrollado su proceso de emprendimiento, y tienen como características principales las siguientes:

- Contar con procesos de formalización

- Demostrar crecimiento empresarial

- Mantener mínimo 5 años en el mercado.

Al emplear el método inductivo, se pretende conocer la realidad de las empresas análisis para describir de forma detallada cada uno de los interrogantes planteados y mediante el análisis cualitativo contrastar con información y artículos relacionados con la utilización de software libre en las empresas.

El impacto de este estudio tendrá efecto positivo en la sociedad, dado que permitirá brindar conocimientos sobre las ventajas y desventajas del software libre, relacionada con la importancia de los avances tecnológicos en la transformación del desarrollo económico en el contexto empresarial, a partir de estrategias de crecimiento empresarial, formación de capital intelectual, transformación del estado, políticas de emprendimiento y la competitividad.

Los sistemas de información son actualmente uno de los factores clave en el desarrollo y competitividad de la empresa. El Software Libre es una solución tecnológica de alta calidad que ofrece profundas ventajas para que las empresas accedan a las TIC de última generación, equilibrando la desigualdad de recursos entre las pequeñas y grandes empresas. (CESLCAM, 2011)

Tal como lo indica este artículo tecnológico, las cualidades del Software Libre se reflejan en el uso cada vez más mayoritario de grandes empresas y administraciones públicas de todo el mundo: IBM, HP, Nasa, Google, Telefónica, Gobierno de EEUU, Alemania, Francia, España.

El tema del software libre está muy relacionado con el emprendimiento, obviamente crear empresa implica una inversión alta y el mundo del software libre pretende ser un aliciente para estas empresas que comienzan, pero irónicamente grandes empresas y entidades públicas en el mundo, como la NASA, Michelin \& Samsung entre otras, trabajan con aplicaciones como Open Suse o Ubuntu. El tema libre ha sido asumido como una filosofía y por su puesto un modelo de solidaridad en torno a la creación de sistemas y herramientas gratuitos para la humanidad. (Mendivil, 2013)

El emprendimiento, la innovación y la tecnología van muy de la mano en nuestra Sociedad del Conocimiento. Ejemplo de esto es el Campus Party, que abre un importante espacio para fomentar y apoyar de manera decidida a todos los campuseros que han decidido emprender con una base tecnológica.

Casos de éxito locales y regionales, intercambio de experiencias, networking, talleres de acompañamiento y formación, visibilización de proyectos en desarrollo y acercamiento a inversionistas y capitales riesgo son algunos de los elementos que tendrán a su alcance los emprendedores tecnológicos.

\section{DESARROLLO}

Las empresas de base tecnológica del Departamento del Meta no podrían emprender, sus negocios, si no contaran con un capital inicial destinado a adquirir software legal o licenciado. Por lo cual el software libre nace como una oportunidad de que los emprendimientos tecnológicos puedan desarrollarse con bajo capital e ir creciendo rápidamente.

Shapero presenta cuatro aspectos importantes para que los emprendimientos surjan de forma más sólida, sin decir que solo son las únicas variables que se tienen en cuenta. Entre los aspectos presenta el desplazamiento, disposición a actuar, credibilidad y disponibilidad de recursos (Kantis, 2001), este último contiene elementos como dinero, infraestructura, equipos, materia prima, contactos entre otros, que para el caso de los emprendimientos de base 
tecnológica son indispensables para iniciar su empresa.

En las empresas de base tecnológica se identifican tres procesos importantes del negocio, el gerencial, contable, comercial, y el de producción, para el cual se deben incluir no solo costos de los equipos y la infraestructura, sino también como los plantea (Raúl Francisco Oltra Bandenes, 2010) los costos de licencias, implantación, asesorías y consultorías, formación entre otros.

Es así que las empresas de base tecnológica centran sus esfuerzos en la producción, definiendo este como el proceso para el desarrollo de software, diseño, modelación y simulación de programas, sistemas o aplicaciones que harán parte del portafolio de productos y servicios que estos comercializaran como estrategia de crecimiento para sus empresas y negocios.

Las empresas y la información están a la distancia de un clic, cada día las organización y los negocios fluyen a través de la red, donde el acceso a los programas, educación, códigos fuente, comunidades de trabajo colaborativo y porque no el software pirata, cada vez son más fáciles de acceder a la velocidad del deseo. Es aquí donde la idea de aprovechar estos recursos disponibles, son oportunidades para el desarrollo de ideas y negocios.

\section{ANÁLISIS}

Al entrevistar las empresas de base tecnológica dedicadas al desarrollo de programas, aplicaciones y software en el Departamento del Meta, que en adelante mencionaremos como EBT, se encontró la siguiente información.

En su mayoría las EBT, son formalizadas, pasando por un proceso de transformación de largo plazo. Estas empresas nacen informales, luego son registradas como establecimientos de comercio con el fin mantener un nivel de formalización empresarial que les permita respaldar mediante un nombre comercial, sus actividades; al alcanzar un nivel de madurez empresarial estas pasan a ser personas jurídicas, inclinándose por las Sociedades por acciones simplificadas (S.A.S) que les permite involucrar a los demás miembros de su compañía y tener un estatus que genere seguridad entre sus clientes y proveedores.
Este procesos de crecimiento de las empresas y de transformación se logra en el largo plazo, en promedio las empresas llevan en el mercado 7 años, en los cuales han dedicado entre uno y dos años a establecer el modelo de negocio, a generar redes de trabajo y a fortalecer sus conocimientos y su objeto comercial, a partir de este año deciden invertir en la formalización, a partir de este cambio se demoran dos años para constituirse en sociedades e iniciar una etapa empresarial más seria.

En cuanto al capital semilla o capital inicial que emplean las EBT, se puede determinar que invierten entre 5 y 6 millones de pesos, aportados por tres o cuatro socios fundadores, representados en equipos de cómputo, software y mobiliario de oficina.

(Capital inicial en las empresas)

Tabla 1. Destinación de capital inicial en las EBT.

\begin{tabular}{|l|l|}
\hline Destino & Porcentaje \\
\hline Software & $10 \%$ \\
\hline Hardware & $70 \%$ \\
\hline Instalaciones & $20 \%$ \\
\hline Aspectos legales & $0 \%$ \\
\hline
\end{tabular}

Fuente: Autores

En la tabla vemos como la adquisición de equipos y la inversión en instalaciones tienen un peso importante en la destinación de estos recursos iniciales, solo el $10 \%$ del capital inicial fue destinado a software, lo da origen a la pregunta ¿Cómo hacen entonces estas empresas (EBT) para producir servicios si no cuentan con software para desarrollarlos?

El software con el que cuentan las empresas de base tecnológica en el departamento del Meta, se concentra de cuatro formas diferentes, entre estas, software licenciado o propietario, software de código abierto, software libre, software pirata.

Para el caso del Departamento del Meta la utilización del software se encuentra distribuida como veremos en la tabla 2.

Es claro que para las EBT, el proceso de producción es uno de los más importantes, para el desarrollo de su emprendimiento, por esta razón el $65 \%$ del software que utilizan para la producir, es de código abierto y libre, en este proceso también se emplea el $20 \%$ del software pirata, ya que los emprendedores no cuentan con capital semilla suficiente para iniciar sus labores empresariales. 
Gráfico 1. Característica del software con el que cuentan las EBT

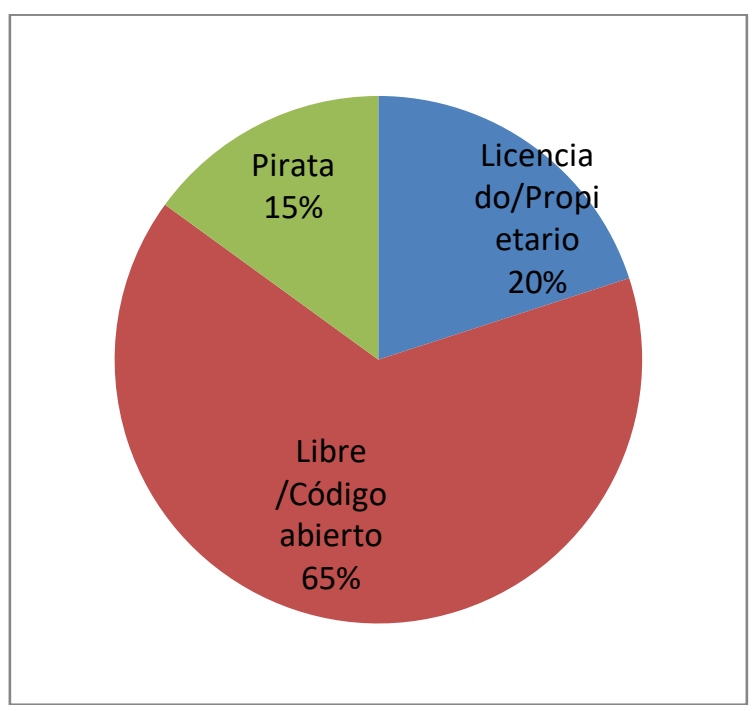

Fuente: Autores

El software con el que cuentan los emprendedores está compuesto por código libre y código abierto en un $65 \%$ y tan solo el $20 \%$ de este es licenciado o propietario. Sin embargo es importante destacar que el $15 \%$ del software es pirata.

Tabla 2. Software destinado a procesos.

\begin{tabular}{|l|c|c|c|c|}
\hline $\begin{array}{l}\text { Tipo de } \\
\text { sofitware }\end{array}$ & $\begin{array}{l}\text { PROD } \\
\text { UC }\end{array}$ & $\begin{array}{l}\text { COMIRC } \\
\text { IAL }\end{array}$ & $\begin{array}{l}\text { CONTAB } \\
\text { LE }\end{array}$ & $\begin{array}{l}\text { GERENCI } \\
\text { AL }\end{array}$ \\
\hline $\begin{array}{l}\text { Licencia } \\
\text { do/ } \\
\text { Propieta } \\
\text { rio }\end{array}$ & $10 \%$ & $70 \%$ & $10 \%$ \\
\hline $\begin{array}{l}\text { Libre/ } \\
\text { Código } \\
\text { abierto }\end{array}$ & $65 \%$ & $100 \%$ & $30 \%$ & $80 \%$ \\
\hline Pirata & $20 \%$ & $100 \%$ & $100 \%$ & $100 \%$ \\
\hline Total & $100 \%$ & $100 \%$ \\
\hline
\end{tabular}

Fuente: Autores

La dedicación al proceso de producción hace que los empresarios dediquen pocos esfuerzos en la comercialización de los productos; en este proceso comercial se destaca que al iniciar los emprendimientos estos emplear software libre y de código abierto para conseguir negocios, hacer contactos entre otros. Para esto emplean redes sociales, foros, paquetes gratuitos de CRM, entre otros.
Para los procesos contables y financieros, los emprendedores utilizan en un $70 \%$ software licenciado, aunque en los inicios de las empresas este procesos se realiza de manera informal, luego de dos años, su contabilidad pasa a tener una estructura más legal por lo que deben apoyarse en contadores que en estos casos son los dueños de las licencias contables que ponen a servicio de los empresarios.

Para los sistemas de ERP que hacen parte de los procesos gerenciales de las EBT, estas emplean en un $80 \%$ software libre, en conjunto para buscar o hacer desarrollos colaborativos, encontrar proveedores, hacer seguimiento a metas o para la planeación.

Es así que el software libre se ha convertido en una ventaja potencial de las pequeñas empresas acelerando e integrando procesos de innovación.

Las iniciativas empresariales, necesitan disponer de las aplicaciones necesarias para enfrentar los retos de la producción y modernización (Parada, 2011), que le permitan estar a la vanguardia de los adelantos, actualizaciones, mejoras y publicaciones de software, que estos empresarios puedan emplear para el desarrollo de sus negocios.

El software libre no solo le ofrece a los emprendedores la posibilidad de desarrollar sus ideas de negocios, sino que se convierte en gran parte de la sostenibilidad del negocio. Tanto así que el solo hecho de requerir modificaciones de códigos, requieren personal capacitado que adapte esas tecnologías a las necesidades de su empresa para poder producir más software que a su vez generara nuevos códigos requeridos por nuevos usuarios, así los sistemas se van robusteciendo y actualizándose a medida que pasa el tiempo, adaptándose a las necesidades del mercado y la empresa.

En este sentido los EBT producen desarrollos de software a la medida para que sean adquiridos por empresas que se verán en encrucijadas éticas en el momento de adquirir estos productos, por el hecho de no poder hacer trazabilidad al código y conocer el grado plagio que pueda tener el software que están adquiriendo, es aquí donde las empresas evalúan la adquisición de software en cuatro factores.

- Responsabilidad: determina quien se hace cargo si el software falla.

- Proceso de aprendizaje: El instalar software libre conlleva un proceso de aprendizaje 
- Relación Costo - Calidad: no siempre los productos caros son mejores.

- Solidez empresarial: Se le atribuye a las empresas de software libre la condición de inestables aunque no es así. (José Ramón Pérez Agüera, 2004).

\section{CONCLUSIONES}

Las EBT, en general tienen como principal objetivo la producción de software y aplicaciones, basándose en herramientas de software libre y herramientas de código abierto, debido a que nacen informales y no se preocupan por adquirir software para la gestión comercial y empresarial en sus inicios. El hecho de que las empresas de desarrollo de software sean en sus iniciaos informales y la mayoría de sus procesos de producción de software se haga basado en herramientas de código abierto y software libre, no quiere decir que sus productos sean de mala calidad o que su seguridad sea baja.

La existencia en la nube de aplicaciones, software, código y redes colaborativas de trabajo, hacen que los emprendimientos de base tecnológica tengan futuro en el mercado, ya que son materia prima para producción de software, sin estos software las empresas no podrían iniciar sus actividades, ya que el capital para iniciar legalmente es alto.

Aunque la piratería de software siempre será un tema ético en las compañías, para las empresas de desarrollo de software pequeñas que son resultados de esfuerzos iniciados en la universidad, esta tendencia tiende a desaparecer a medida que las empresas van creciendo y consolidando en el mercado, ya que es el mercado quien lo regula ética y moralmente.

\section{BIBLIOGRAFÍA}

CESLCAM, C. d.-L. (04 de Octubre de 2011). Centro de Apoyo Tecnológico a Emprendedores.

Escalante, Z. \&. (2009). Modelos de negocios en entornos de desarrollo tecnologico. Centro de investigación de ciencias administrativas y gerenciales CICAG, 102.
José Ramón Pérez Agüera, R. S. (2004). Documentación y Software Libre. Universidad Complutense de Madrid, 41-49.

Kantis, H. A. (2001). Nuevos emprendimientos y emprendedores: de qué depende su creación y supervivencia. Explorando el caso argentino,. UNGS-LITTEC, DT, 2., 30.

Mendivil, I. R. (2013). Colombia Aprende . Obtenido de

http://www.colombiaaprende.edu.co/html/home/1592 /article-170660.html

Parada, C. G. (2011). La localizacion de aplicaciones de software libre en el ámbito de las empresas. Revista Tradumática, 108-117.

Raúl Francisco Oltra Bandenes, H. G. (2010). Factores diferenciasles entreo los ERP de software libre (FSw ERP) y los ERP propietarios. Valencia: Universidad Politécnica de Valencia. 